\title{
A fast, simple, and reliable hydrophilic interaction liquid chromatography method for the determination of ascorbic and isoascorbic acids
}

\author{
Ana I. R. N. A. Barros • Ana P. Silva • \\ Berta Gonçalves $\cdot$ Fernando M. Nunes
}

Received: 11 October 2009 /Revised: 14 December 2009/Accepted: 16 December 2009/Published online: 21 January 2010

(C) Springer-Verlag 2010

\begin{abstract}
A reliable method for the determination of total vitamin $\mathrm{C}$ must be able to resolve ascorbic acid (AA) and the epimeric isoascorbic acid (IAA) and determine the sum of AA and its oxidized form dehydroascorbic acid. AA and IAA are polar molecules with a low retention time in conventional reversed phase systems, and hence of difficult resolution. Hydrophilic interaction chromatography using a TSKgel Amide-80 stationary phase with isocratic elution was successful in resolving the two epimers. The column was compatible with injections of high concentrations of metaphosphoric acid, tris(2-carboxyethyl)-phosphine, and EDTA without drift of baseline and retention time. Total $\mathrm{AA}$ and IAA were extracted, stabilized, and reduced in one step at $40{ }^{\circ} \mathrm{C}$, using $5 \% \mathrm{~m}$-phosphoric acid, $2 \mathrm{mM}$ of EDTA, and $2 \mathrm{mM}$ of tris(2-carboxyethyl)-phosphine as reducing agent. This simple, fast, and robust hydrophilic interaction chromatography-DAD method was applied for
\end{abstract}

\footnotetext{
A. I. R. N. A. Barros • F. M. Nunes $(\bowtie)$

CQ - Chemistry Research Centre, Chemistry Department,

University of Trás-os-Montes e Alto Douro,

5000-801 Vila Real, Portugal

e-mail: fnunes@utad.pt

A. P. Silva

CITAB - Centre for the Research and Technology

of Agro-Environment and Biological Sciences,

Department of Agronomy,

University of Trás-os-Montes e Alto Douro,

5001-801 Vila Real, Portugal

B. Gonçalves

CITAB - Centre for the Research and Technology

of Agro-Environment and Biological Sciences.

Department of Biology and Environment,

University of Trás-os-Montes e Alto Douro,

5001-801 Vila Real, Portugal
}

the analysis of food products namely fruit juices, chestnut, and ham and also in pharmaceutical and multivitamin tablets. Method validation was performed on the food products, including parameters of precision, accuracy, linearity, limit of detection, and quantification (LOQ). The absence of matrix interferences was assessed by the standard addition method and Youden calibration. The method was fast, accurate, and precise with a $\mathrm{LOQ}_{\mathrm{AA}}$ of $1.5 \mathrm{mg} / \mathrm{L}$ and LOQ $_{\text {IAA }}$ of $3.7 \mathrm{mg} / \mathrm{L}$. The simple experimental procedure, completed in $1 \mathrm{~h}$, the possibility of using IAA as an internal standard, and low probability of artifacts are the major advantages of the proposed method for the routine determination of these compounds in a large number of samples.

Keywords Hydrophilic interaction chromatography Vitamin C · Ascorbic acid · Isoascorbic acid

\section{Introduction}

L-Ascorbic acid (AA; Fig. 1 I), together with its oxidized form dehydroascorbic acid (DHA; Fig. 1 II), makes up the total vitamin $\mathrm{C}$ content of foods. It is a water-soluble vitamin with antioxidant properties [1] and a range of other important biological functions [2], with fruits and vegetables being the main dietary source [3]. Both AA and DHA are absorbed through the gastrointestinal tract [4] and can be interchanged enzymatically in vivo [5]. The current recommended daily allowance for vitamin $\mathrm{C}$ is determined as $60 \mathrm{mg}$ /day [6]. As an antioxidant vitamin, AA is used in multi-component vitamin preparations, analgesics, being also used for enrichment of foods and drinks to increase its vitamin $\mathrm{C}$ content and for labeling purposes. AA is also an important food additive (European Union Food Additive $\mathrm{E}$ number E300 [7]), where it is used as an antioxidant for the 


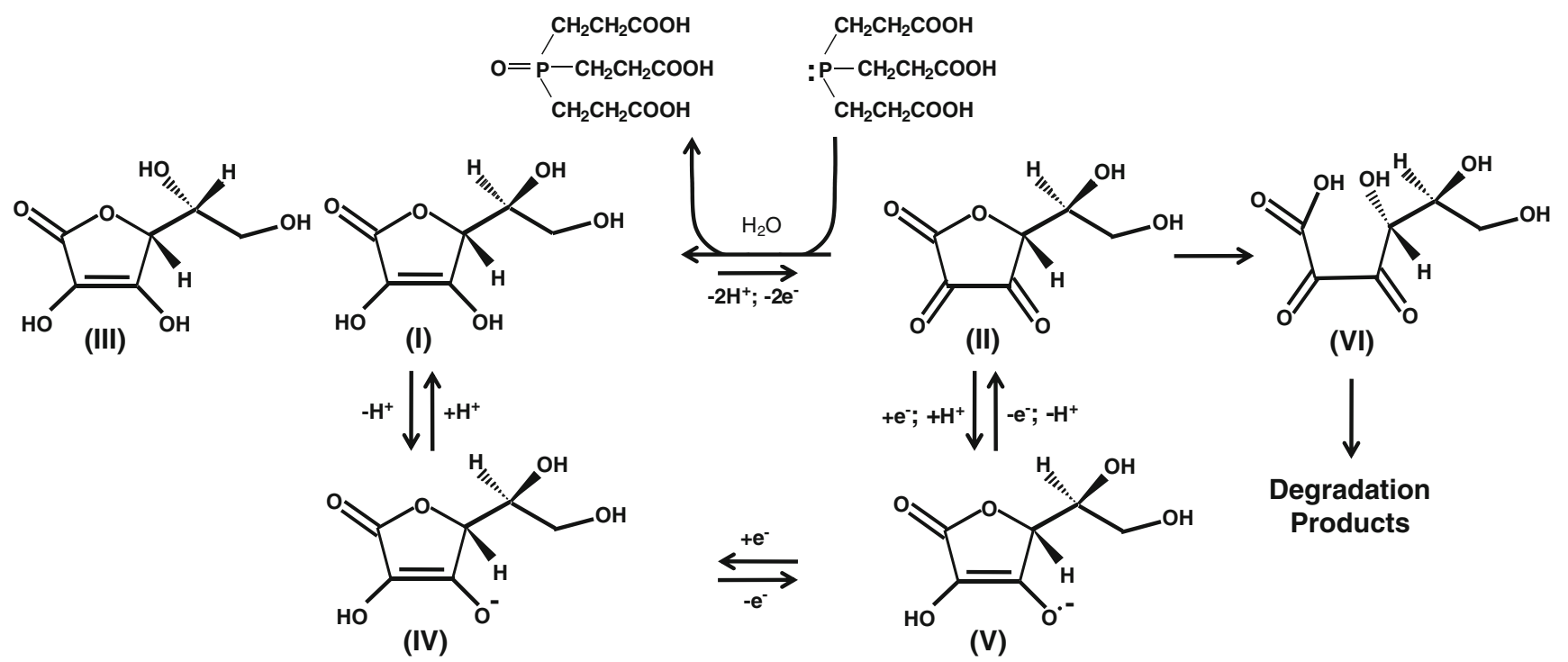

Fig. 1 Chemical structures of ascorbic acid (I), dehydroascorbic acid (II), and isoascorbic acid (III) and reaction pathways and degradation

processing of beverages, wines, and meat products. With the same purpose, isoascorbic acid (IAA, Fig. 1 III), the $\mathrm{C}_{5}$ epimer of AA (D-ascorbic acid or erythorbic acid), is also frequently used (E315 [9]). Although for food applications, IAA has the same antioxidant activity of AA, and IAA has much lower vitamin $\mathrm{C}$ activity (nearly $5 \%$ ) [8].

A reliable method for the determination of vitamin $\mathrm{C}$ in food products needs to be able to quantify AA and DHA and be able to differentiate IAA. Quantification of AA in foods is routinely performed by the indophenol titration method [9] or the fluorescence quantification of the quinoxaline derivatives of DHA, formed after oxidation of AA and reaction with $o$-phenylenediamine [10]. These methods will not distinguish between AA and IAA and cannot be used for quantification of vitamin $\mathrm{C}$ in a range of foods containing IAA like processed and cured meats. Also the reduction of indophenol is not limited to AA, and other reducing substances present in the sample (e.g., cuprous and ferrous ions, tannins, cysteine, and glutathione) can reduce the dye and lead to high measurements [11].

To circumvent these problems, a number of other analytical methods have been developed to quantify the AA that include enzymatic, spectrophotometric, flow injection, electrochemical, gas chromatography, and highperformance liquid chromatography (HPLC) [12]. HPLC methods were mostly used (for a review, see $[13,14]$ ). Total vitamin $\mathrm{C}$ determination can be performed by independent quantification of AA and DHA with a different combination of pre- or post-column derivatization $[15,16]$. These approaches are time consuming, and AA can be degraded during the derivatization procedure. The degradation of AA to DHA and the irreversible hydrolyzes to biologically inactive 2,3-diketo-L-gulonic acid (Fig. $1 \mathrm{~V}$ ) is the most common artifact in vitamin $\mathrm{C}$ analysis. AA can be oxidized through one- or two-electron transfers (Fig. 1). Oneelectron reductions utilize the transition through the AA free radical (semidehydroascorbic acid or monodehydroascorbic acid; Fig. 1 IV). The AA free radical suffers dismutation forming an equimolar mixture of $\mathrm{AA}$ and DHA. Two-electron reductions can occur when transition metals catalyze AA oxidation directly to DHA. Factors that affect the AA stability in solution include light, temperature, $\mathrm{pH}$, the presence of oxygen and metal ions, and even possible ascorbic acid oxidase enzymes in biological extracts [12]. To prevent oxidation of AA, stabilizing solutions such as $m$-phosphoric acid (MPA) were used for extractions $[12-14,20]$. MPA inhibits metal catalysis and ascorbic oxidase and precipitates proteins aiding in the extract clarification [17]. Another approach is using reducing agents, which in addition to inhibit oxidation, are also employed to convert DHA to AA, and determine their sum: the total vitamin $\mathrm{C}$ as one component. Tris(2-carboxyethyl) phosphine hydrochloride (TCEP) offers an efficient reduction of DHA at an acidic $\mathrm{pH}[18,19]$. The determination of total vitamin $\mathrm{C}$ by using the reduced form (AA) allows the use of common detectors such as UV, as DHA gives a low response on the majority of chromatographic detectors.

Different chromatographic approaches have been used to resolve AA and IAA. Conventional reversed phase chromatography (RP-HPLC) does not give a sufficient retention of these very polar molecules, not allowing their total resolution and neither their good separation from the dead volume, even when using no organic solvent in the mobile phase. Also MPA can coelute or interfere with AA or IAA and generating inconsistency in their retention times [20]. These drawbacks can be resolved by using polymeric 
stationary phases as styrene-divinylbenzene columns [21, 22]. Another widely used approach is ion-pairing chromatography [23-26, 38], where also the separation of AA and IAA are attained. Nevertheless, because of the ion-pairing separation principle, no retention is observed in new unequilibrated column, the mobile phase composition is complex, and thorough equilibration before use is needed, and the column lifetime may be limited [26]. Ion-exchange separation of AA and IAA on aminopropyl columns [23, 27, 28, 43] have been carried out, but an on-column oxidation of AA that could not be completely eliminated was observed [23, 29]. No column oxidation is observed on a polymer-coated, silica-based $\mathrm{NH}_{2}$ column [41].

Recently hydrophilic interaction chromatography (HILIC) has been used to perform AA analysis [30-33] using different HILIC stationary phases. Only one of the works addressed the question of AA and IAA resolution [44]. HILIC was found to be adequate for increasing AA and IAA chromatographic retention, but a disadvantage was the need to perform the extractions and standard preparation in solutions with high organic solvent content and buffers used in the mobile phase, limiting the use of stabilizers to those soluble in the organic phase, acetonitrile, which excludes the use of the most effective stabilizer MPA [45]. This limitation is due to the need to provide similarity to the mobile phase used in the chromatographic separation, as changes in concentration of the organic modifier lead to retention time shifts and irregular peak shapes [15, 45]. This limits their accurate quantification due to the AA and IAA instability. If these important issues are resolved, the HILIC approach seems to be a better alternative to the other chromatographic separation modes.

The aims of this work were to develop, optimize, and validate a fast, simple, and reliable method for the determination of AA and IAA based on the HILIC approach capable of overcoming the stability problems presented by the earlier HILIC methods. This was possible using an amide-bonded silica (TSKgel Amide-80). This hydrophilic phase contains a carbamoyl (amide) group on a short aliphatic spacer $[34,35]$, being less reactive than the amine group and without its basicity, showing good recoveries and stability. The method was validated for a range of food matrix including fruit juices, chestnut, and ham. Also the applicability to multivamin tablets and analgesics was addressed.

\section{Experimental}

Chemicals and reagents

Working standards of ascorbic acid (Merck), isoascorbic acid (BDH), and dehydroascorbic acid (Sigma-Aldrich) were used. MPA, tris(2-carboxyethyl)-phosphine hydrochloride (TCEP·HCl; $>98 \%$ ), trofluoroacetic acid, and acetic acid were from (Sigma-Aldrich), and formic acid (pro-analysis) and acetonitrile (HPLC gradient grade) were purchased from Panreac. HPLC-grade water was prepared with a SGS water purification system. The regenerated cellulose membrane filters were from Marchery-Nigel.

\section{Apparatus and chromatographic conditions}

A Dionex liquid chromatographic system (USA) consisting of an Ultimate 3000 pump, PDA-100 photodiode array detector, a WPS-3000TSL Analyt auto sampler, and column oven (Model TCC-3200) were used. The chromatographic analyses were carried out on a TSKgel Amide80 (4.6 i.d. $\times 100$ mm, $5 \mu \mathrm{m}$, Tosoh Bioscience, Japan) kept at $20{ }^{\circ} \mathrm{C}$, unless otherwise stated. The optimal analysis conditions were carried out using acetonitrile (B): aqueous $0.1 \%$ trifluoroacetic acid (A) $(90: 10, v / v)$ as a mobile phase during $7 \mathrm{~min}$, changed to $80 \% \mathrm{~A}$ at $8 \mathrm{~min}$ and maintained until $13 \mathrm{~min}, 10 \% \mathrm{~A}$ at $14 \mathrm{~min}$ and maintained at $10 \% \mathrm{~A}$ until $20 \mathrm{~min}$. The flow rate was $0.7 \mathrm{~mL} / \mathrm{min}$, and the absorbance in the $210-350-\mathrm{nm}$ range was monitored, and the absorbance at $244 \mathrm{~nm}$ was used for analysis.

\section{DHA and IDHA reduction and AA and IAA stability}

The AA and IAA stability was assessed before method development and validation. As AA and IAA are known to be unstable in aqueous solution, the relevant factors that influence their stability were accessed: temperature $\left(4{ }^{\circ} \mathrm{C}\right.$ and room temperature) and the concentration of $\mathrm{AA}$ and IAA in solution $(250,100$, and $25 \mathrm{mg} / \mathrm{L})$. For stabilizing the AA and IAA in solution, a 5\% MPA, 2 mM TCEP, and $2 \mathrm{mM}$ EDTA solution were used. The stability of solutions was assessed immediately after preparation until $48 \mathrm{~h}$.

The kinetics of DHAA reduction by the extraction and reduction solution was followed chromatographically. Three DHA concentrations were used $(25,50$, and $100 \mathrm{mg} /$ L) and three temperatures $\left(20,30\right.$, and $\left.40{ }^{\circ} \mathrm{C}\right)$. Isodehydroascorbic acid (IDHA) was formed by the addition of IAA $(25 \mathrm{mg})$ to DHA $(50 \mathrm{mg})$ in water $(50 \mathrm{~mL})$, and after oxidation of 42 and $78 \mathrm{mg} / \mathrm{L}$ of IAA, the solution was twofold diluted with the reduction solution (10\% MPA, $4 \mathrm{mM}$ TCEP, and $4 \mathrm{mM}$ EDTA), and the recovery of IAA and AA was followed chromatographically.

Optimization of the chromatographic separation

The chromatographic separation was optimized for the composition of the mobile phase by varying the acetonitrile content $(97.5 \%$ to $85 \%$ ), aqueous solution composition in terms of acid used (acetic, formic, and trifluroacetic each 
$0.1 \%$ ), injection volume ( 5 to $100 \mu \mathrm{L}$ ), mobile phase flow rate $(0.5$ to $2.0 \mathrm{~mL} / \mathrm{min})$, and column temperature (10 to $30{ }^{\circ} \mathrm{C}$ in $5{ }^{\circ} \mathrm{C}$ intervals). The optimization parameters used were resolution between AA and IAA peaks, peak area, retention time, efficiency, and peak asymmetry.

Standard preparation and calibration curve

Stock solutions $(2,500 \mathrm{mg} / \mathrm{L})$ were freshly prepared by dissolving AA, IAA, and in the extraction-reduction solution containing 5\% MPA, $2 \mathrm{mM}$ TCEP, and $2 \mathrm{mM}$ EDTA. Standard solutions between 0.5 and $500 \mathrm{mg} / \mathrm{L}$ for $\mathrm{AA}$ and IAA were prepared by diluting the corresponding intermediate stock solution in the extraction-reduction solution. External calibration curves were constructed in the range of $1.0-500 \mathrm{mg} / \mathrm{L}$ by plotting the peak areas of each compound versus concentration and gave the values of slope along with the intercept and correlation coefficient for each calibration curve. Each point was analyzed in quadruplicate.

Internal standard calibration for quantification of AA was performed using IAA as an internal standard. Calibration curves were constructed in the range $1.0-500 \mathrm{mg} / \mathrm{L}$ of AA adding $250 \mathrm{mg} / \mathrm{L}$ of IAA, by plotting the peak areas ratio of AA/IAA versus concentration ratio of AA.

\section{Sample preparation}

For the analysis of fruit juices (orange, pear, and mango bought on a local market), the samples were fivefold diluted (final volume $50 \mathrm{~mL}$ ) with a concentrated extraction-reduction solution (6.25\% MPA, $2.5 \mathrm{mM}$ TCEP, and $2.5 \mathrm{mM}$ EDTA) and heated at $40 \pm 2{ }^{\circ} \mathrm{C}$ for $60 \mathrm{~min}$ in a forced air oven. After filtration through $0.45 \mu \mathrm{m}$ in pore size syringe filter (regenerate cellulose membrane), it was analyzed.

For the analysis of freeze dried and ground chestnuts by external calibration, $5.0 \mathrm{~g}$ of sample was weighted to 50 $\mathrm{mL}$ plastic centrifuged tubes and was extracted with $25 \mathrm{~mL}$ of the extraction-reduction solution (5\% MPA, $2 \mathrm{mM}$ TCEP, and $2 \mathrm{mM}$ EDTA) for $60 \mathrm{~min}$ at $40 \pm 2{ }^{\circ} \mathrm{C}$. After centrifuging for $5 \mathrm{~min}$ at $3,000 \times \mathrm{g}$, the supernatant was transfer to a volumetric flask. The residue was extracted three more times with fresh $25 \mathrm{~mL}$ of the extractionreduction solution for $15 \mathrm{~min}$ at $40 \pm 2{ }^{\circ} \mathrm{C}$ and centrifuged for $5 \mathrm{~min}$ at $3,000 \times \mathrm{g}$, the supernatant was added to the previous ones, and the flask was filled up to the mark with the extraction solution. After filtration through a $0.45-\mu \mathrm{m}$ syringe filter, it was analyzed. For the analysis of freezedried chestnuts by internal standard calibration, after weighting $2.5 \mathrm{~g}$ of chestnuts, $10 \mathrm{~mL}$ of IAA internal standard $(2,500 \mathrm{mg} / \mathrm{L}$ in the extraction-reduction solution) was added, followed by addition of $40 \mathrm{~mL}$ of the extraction-reduction solution, and after standing for $60 \mathrm{~min}$ at $40 \pm 2{ }^{\circ} \mathrm{C}$, the suspension was centrifuged for $5 \mathrm{~min}$ at $3,000 \times \mathrm{g}$, and a sample was taken, filtered, and analyzed.

For the analysis of ham by external calibration, samples $(5 \mathrm{~g})$ were weighted to $50-\mathrm{mL}$ plastic centrifuged tubes, and after adding $25 \mathrm{~mL}$ of the extraction-reduction solution (5\% MPA, $2 \mathrm{mM}$ TCEP, and $2 \mathrm{mM}$ EDTA), the mixture was homogenized in an Ultra-Turrax for $1 \mathrm{~min}$, and the disperser tool was washed with further $10 \mathrm{~mL}$ of the extraction-reduction solution. The dispersion was heated at $40 \pm 2{ }^{\circ} \mathrm{C}$ for $60 \mathrm{~min}$, and it was centrifuged at $3,000 \times \mathrm{g}$ for $15 \mathrm{~min}$. The supernatant was transferred to a volumetric flask, and the residue was extracted two more times with fresh $25 \mathrm{~mL}$ of the extraction-reduction solution for $15 \mathrm{~min}$ at $40 \pm 2{ }^{\circ} \mathrm{C}$ and centrifuged for $15 \mathrm{~min}$ at $3,000 \times \mathrm{g}$, the supernatants were added to the previous ones, and the flask was filled up to the mark with the extraction solution. After filtration through a $0.45-\mu \mathrm{m}$ syringe filter, it was analyzed.

For the analysis of analgesics and multivitamin tablets, one tablet was dissolved in $100 \mathrm{~mL}$ of the extractionreduction solution and kept at $40 \pm 2{ }^{\circ} \mathrm{C}$ for $1 \mathrm{~h}$. After this time, the solution was adequately diluted (twofold for multivitamin tablet and ten times for the analgesic tablets) with the same solution and analyzed. Each product was analyzed six times.

The extraction-reduction solvent was found to be stable for at least 15 days at $4{ }^{\circ} \mathrm{C}$ (at this temperature, a small precipitate is formed that can be re-dissolved after warming to room temperature).

\section{In-house method validation}

The reliability of the proposed method was validated according to the IUPAC, ISO, AOAC harmonized guidelines for single laboratory validation of methods of analysis [36]. Additionally the system suitability was also evaluated, and the system performance limits were those of European Pharmacopoeia [37].

System suitability test and robustness

The system suitability test (SST) was performed under optimized chromatographic conditions. Theoretical plates, peak asymmetry, resolution of individual compounds, and the repeatability of reference standard solution injections have been established (retentions times and peak areas were checked), made in ten replicates.

The robustness of the HPLC method was evaluated by small variations in the method parameters shown in the method optimization, to influence decisively the performance parameters: acetonitrile percentage $(91 \%$ and $89 \%)$, trifluoroacetic (TFA) concentration $(0.09 \%$ and $0.11 \%)$, 
flow rate $(0.6$ and $0.8 \mathrm{~mL} / \mathrm{min})$, and column temperature $\left(17\right.$ and $23{ }^{\circ} \mathrm{C}$ ). A full factorial design was used, and the different conditions were analyzed randomly. The system was stabilized by running five times the studied conditions, and only the last two were used for calculation.

Linearity, detection, and quantification limits

Linearity was evaluated through the relationship between the concentration of AA or IAA for the external calibration of between the concentration ratio AA for the internal standard calibration (independent variable) and the absorbance obtained after analysis by HPLC and detection by DAD (dependent variable) according to the ISO 8466-1 [38]. For determination of detection limit, a calibration curve covering the lower range of the calibration curve $(0.5-1.0-2.5-5.0-7.5-10-25 \mathrm{mg} / \mathrm{L})$ was analyzed in quadruplicate in random order, and the detection limit was calculated as described elsewhere [39].

\section{Recovery, precision, and selectivity}

Recovery was tested by spiking the different matrixes with known amounts of AA or IAA acids at three levels $(25$, 250 , and $500 \mathrm{mg} / \mathrm{mL}$ ) and analyzing six times the unfortified and fortified test portions. The intra-day variation was determined by assaying ten replicates on the same day of the sample under study, and inter-day variation was assayed for quadruplicates on 3 days. The precision was expressed as the percentage of relative standard deviation (\%RSD). Method selectivity was assessed by comparing the UV spectrum in the standards with those obtained in the real samples. The peak purity match factor calculated by the HPLC software (Chromeleon) was also assessed.

Testing general matrix effect—-standard addition and Youden calibration

The possible effects of the different food matrixes studied in the AA or IAA response a standard addition calibration and Youden calibration were performed [40, 41]. Standardadded calibration (SAC) was obtained by addition of different amounts of AA or IAA standard solution (500 to
$25 \mathrm{mg} / \mathrm{L}$ ) to a constant volume or amount of sample of the standard protocol, performed in duplicate. The zero addition was also included. The samples were treated as previously mentioned and analyzed by the proposed HILIC method. Linear least square regression of analytical signal versus amount of AA or IAA added for each matrix were established, and slope, intercept, and residual standard deviation for each curve were calculated. A Student's $t$ test was applied to compare the slope obtained by SAC and external calibration or internal standard calibration.

For the Youden calibration, different amounts of the sample were measured (chestnuts and ham, 10 to $1.0 \mathrm{~g}$; fruit juices, 25 to $5 \mathrm{~mL}$ ), maintaining the final extractionreduction concentration constant. Each sample was analyzed in duplicate by the standard protocol applied for each kind of sample. Youden calibration curves were constructed by plotting the peak areas of each compound versus the weight or volume of the sample analyzed and gave the values of slope along with the intercept and correlation coefficient for each Youden calibration curve. A Student's $t$ test was applied for detecting an intercept obtained by Youden calibration significantly different from zero.

\section{Results and discussion}

\section{Optimization of the HILIC method}

With the purpose of developing a fast and efficient HILIC method for the quantification of AA and IAA, a short column $(10 \mathrm{~cm})$ of amide-bonded silica (TSKgel Amide80) was chosen as stationary phase. Acetonitrile has been shown to be most adequate organic solvent for HILIC [42] and was used without further investigation. Other variables that influence the retention on HILIC mode were subjected to optimization, namely, the acetonitrile/aqueous solution ratio used as mobile phase ( $85 \%$ to $97.5 \%$ of acetonitrile), the use of an acidified aqueous solution $(0.1 \%$ of acid and no acid), the type of acid used (TFA and acetic and formic acid), the column temperature $\left(10\right.$ to $30{ }^{\circ} \mathrm{C}$ ), and mobile phase flow rate $(0.5$ to $2.0 \mathrm{~mL} / \mathrm{min})$. For each chromatographic condition, the retention time of AA and IAA, resolution, peak asymmetry, plate number, and peak area

Table 1 Effect of the mobile phase composition (\% acetonitrile) on the chromatographic separation of AA and IAA

\begin{tabular}{|c|c|c|c|c|c|c|c|c|c|}
\hline & $\mathrm{Rt}_{\mathrm{IAA}}$ & $\mathrm{Rt}_{\mathrm{AA}}$ & Area $_{\text {IAA }}$ & Area $_{\mathrm{AA}}$ & Asym $_{\text {IAA }}$ & Asym $_{\mathrm{AA}}$ & $N_{\mathrm{IAA}}$ & $N_{\mathrm{AA}}$ & Rs \\
\hline $85 \%$ & $3.49 \pm 0$ & $4.04 \pm 0$ & $188.6 \pm 0.3$ & $184.5 \pm 0.1$ & $1.11 \pm 0.01$ & $1.03 \pm 0.01$ & $984 \pm 8$ & $1,195 \pm 11$ & $1.21 \pm 0.01$ \\
\hline $87.5 \%$ & $3.85 \pm 0.005$ & $4.62 \pm 0$ & $189.1 \pm 0.2$ & $183.6 \pm 0.4$ & $1.06 \pm 0.02$ & $1.04 \pm 0.01$ & $757 \pm 4$ & $1,008 \pm 6$ & $1.34 \pm 0.01$ \\
\hline $90 \%$ & $4.44 \pm 0.02$ & $5.60 \pm 0.04$ & $189.3 \pm 0.4$ & $182.9 \pm 0.2$ & $1.00 \pm 0.02$ & $1.00 \pm 0.03$ & $583 \pm 7$ & $804 \pm 8$ & $1.52 \pm 0.02$ \\
\hline $92.5 \%$ & $5.41 \pm 0$ & $7.27 \pm 0$ & $189.9 \pm 0.7$ & $182.3 \pm 0.9$ & $0.88 \pm 0.01$ & $0.89 \pm 0.01$ & $432 \pm 8$ & $712 \pm 14$ & $1.74 \pm 0.02$ \\
\hline $95 \%$ & $7.54 \pm 0.01$ & $11.17 \pm 0.02$ & $190.0 \pm 0.3$ & $181.3 \pm 0.2$ & $0.77 \pm 0.01$ & $0.85 \pm 0.01$ & $356 \pm 1$ & $659 \pm 13$ & $2.18 \pm 0.01$ \\
\hline
\end{tabular}


Table 2 Effect of the mobile phase flow rate $(\mathrm{mL} / \mathrm{min})$ on the chromatographic separation of AA and IAA

\begin{tabular}{|c|c|c|c|c|c|c|c|c|c|}
\hline & $\mathrm{Rt}_{\mathrm{IAA}}$ & $\mathrm{Rt}_{\mathrm{AA}}$ & Area $_{\mathrm{IAA}}$ & Area $_{\mathrm{AA}}$ & Asym $_{\text {IAA }}$ & Asym $_{\mathrm{AA}}$ & $N_{\mathrm{IAA}}$ & $N_{\mathrm{AA}}$ & Rs \\
\hline 0.50 & $6.26 \pm 0.23$ & $7.92 \pm 0.41$ & $278.1 \pm 2.9$ & $271.8 \pm 2.6$ & $0.96 \pm 0.02$ & $0.93 \pm 0.02$ & $548 \pm 22$ & $841 \pm 31$ & $1.53 \pm 0.07$ \\
\hline 0.70 & $4.44 \pm 0.03$ & $5.62 \pm 0.06$ & $191.2 \pm 0.7$ & $184.7 \pm 1.5$ & $1.01 \pm 0.03$ & $1.02 \pm 0.02$ & $571 \pm 12$ & $795 \pm 14$ & $1.55 \pm 0.02$ \\
\hline 1.00 & $3.10 \pm 0.04$ & $3.92 \pm 0.10$ & $139.8 \pm 0.3$ & $137.6 \pm 0.3$ & $0.97 \pm 0.01$ & $0.96 \pm 0.01$ & $508 \pm 17$ & $774 \pm 19$ & $1.47 \pm 0.02$ \\
\hline 1.20 & $2.60 \pm 0.04$ & $3.28 \pm 0.06$ & $117.2 \pm 1.2$ & $114.9 \pm 1.2$ & $0.96 \pm 0.01$ & $0.95 \pm 0.02$ & $533 \pm 1$ & $858 \pm 18$ & $1.51 \pm 0.02$ \\
\hline 1.50 & $2.08 \pm 0$ & $2.62 \pm 0.01$ & $94.4 \pm 0.1$ & $92.6 \pm 0.4$ & $0.96 \pm 0.01$ & $0.97 \pm 0.02$ & $517 \pm 13$ & $833 \pm 1$ & $1.50 \pm 0.01$ \\
\hline 1.75 & $1.78 \pm 0.01$ & $2.24 \pm 0.01$ & $80.7 \pm 0.1$ & $79.2 \pm 0.1$ & $0.96 \pm 0.01$ & $0.98 \pm 0.01$ & $521 \pm 3$ & $814 \pm 4$ & $1.49 \pm 0.01$ \\
\hline 2.00 & $1.55 \pm 0.01$ & $1.96 \pm 0.05$ & $70.5 \pm 0.2$ & $69.1 \pm 0.2$ & $0.98 \pm 0.02$ & $0.97 \pm 0.01$ & $516 \pm 1$ & $799 \pm 23$ & $1.48 \pm 0.01$ \\
\hline
\end{tabular}

were used as output optimization variables. The use of $97.5 \%$ of acetonitrile as mobile phase was excluded in the initial survey as it resulted in large retention times ( $>12 \mathrm{~min})$ and broad peaks. The results obtained for the varying amounts of acetonitrile (containing 0.1\% TFA aqueous solvent as A) are shown in Table 1. Increasing amounts of acetonitrile lead to an increase in retention of both IAA and AA as expected [34, 42]. The resolution also increases with increasing acetonitrile composition, exceeding 1.5 for the $90 \%$ acetonitrile mobile phase composition. The asymmetry of IAA and AA peaks decreases with increasing acetonitrile content, reaching the value of 1 for $90 \%$ acetonitrile. The $90 \%$ acetonitrile mobile phase was used in flow rate optimization.

Table 2 shows the effect of flow rate on the separation of AA and IAA. For a flow rate of $2.0 \mathrm{~mL} / \mathrm{min}$, the retention times of IAA and AA were 1.55 and $1.96 \mathrm{~min}$, respectively, and the resolution is near 1.5. Under these conditions, IAA and AA can be well resolved in nearly $2 \mathrm{~min}$, allowing a faster separation when compared to the common ion-pair RP-HPLC methods traditionally used [16]. Nevertheless, as the flow rate increases, peak areas for AA and IAA decrease significantly. At $2.0 \mathrm{~mL} / \mathrm{min}$, the peak area of AA and IAA was only $29 \%$ of that obtained with a flow rate of $0.5 \mathrm{~mL} /$ min. As higher flow rates decreased significantly the sensitivity, the flow rate $0.7 \mathrm{~mL} / \mathrm{min}$ was chosen as a good compromised between sensitivity and time of analysis. Although at $0.7 \mathrm{~mL} / \mathrm{min}$ the sensitivity was $69 \%$ of that at $0.5 \mathrm{~mL} / \mathrm{min}$, the time of analysis was decreased by $30 \%$.

Traditionally in the HILIC separation mode, the aqueous phase is acidified or buffered on a suitable $\mathrm{pH}[34,42]$. The acidification or buffering is intended to confer the proper charge to the compounds being analyzed and eliminate the residual negatively charged sites on the silica-based stationary phase, affecting retention and improving the peak shape. In the analysis of IAA and AA, the mobile phase $\mathrm{pH}$ control is even more important, as the stability of $\mathrm{AA}$ and IAA is known to be $\mathrm{pH}$ dependent [13-15]. Also due to the acidic nature of IAA and AA (Fig. 1), the mobile phase $\mathrm{pH}$ can also be used to control the IAA and AA ionization. Different acids were used as aqueous additives, namely, trifluoroacetic (TFA), formic and acetic acid at $0.1 \%$, and water without any additive. The results obtained are shown in Table 3. As the strength of the acid used decreased, the retention time of AA and IAA increased, but the increase in the retention time of AA was higher, resulting in a higher resolution when the acetic and formic acids were used for acidification when compared to TFA. Nevertheless the peak symmetry decreased with decreasing acid strength, although they were acceptable. Also a decrease in the plate number was observed when using the acetic and formic acids, especially for IAA, when compared to TFA. No significant difference in sensitivity was observed for the different acids used. When only water was used, there was also an increase in the retention time of both AA and IAA when compared to TFA, an increase in resolution, and a decrease in peak symmetry. The peak areas obtained for IAA and AA using only water was lower than that obtained when using acidified aqueous solutions, suggesting that on-column degradation of IAA and AA occurred. Due to the complex and uncontrolled nature of the real samples intended to be analyzed by this method, an aqueous solution containing $0.1 \%$ TFA was chosen as the aqueous solvent due to the stronger acidity of TFA.

Table 3 Effect of the aqueous phase composition (type of acid) on the chromatographic separation of AA and IAA

\begin{tabular}{|c|c|c|c|c|c|c|c|c|c|}
\hline & $\mathrm{Rt}_{\mathrm{IAA}}$ & $\mathrm{Rt}_{\mathrm{AA}}$ & Area $_{\text {IAA }}$ & Area $_{\mathrm{AA}}$ & Asym $_{\text {IAA }}$ & $\operatorname{Asym}_{\mathrm{AA}}$ & $N_{\text {IAA }}$ & $N_{\mathrm{AA}}$ & Rs \\
\hline TFA & $4.44 \pm 0.02$ & $5.60 \pm 0.04$ & $192.1 \pm 0.6$ & $186.0 \pm 0,8$ & $1.00 \pm 0.02$ & $1.00 \pm 0.03$ & $583 \pm 15$ & $804 \pm 21$ & $1.52 \pm 0.04$ \\
\hline Formic & $4.81 \pm 0.05$ & $6.23 \pm 0.08$ & $191.4 \pm 0.9$ & $186.5 \pm 1.0$ & $0.98 \pm 0.02$ & $0.95 \pm 0.02$ & $488 \pm 7$ & $754 \pm 10$ & $1.60 \pm 0.02$ \\
\hline Acetic & $4.87 \pm 0.07$ & $6.31 \pm 0.12$ & $193.6 \pm 1.0$ & $186.7 \pm 0.8$ & $0.95 \pm 0.01$ & $0.94 \pm 0.01$ & $476 \pm 14$ & $751 \pm 12$ & $1.60 \pm 0.02$ \\
\hline Water & $4.70 \pm 0.05$ & $5.93 \pm 0.02$ & $189.9 \pm 0.3$ & $182.1 \pm 0.3$ & $0.85 \pm 0.01$ & $0.85 \pm 0.01$ & $626 \pm 7$ & $955 \pm 5$ & $1.63 \pm 0.02$ \\
\hline
\end{tabular}


Table 4 Column temperature effect on the chromatographic separation of AA and IAA

\begin{tabular}{|c|c|c|c|c|c|c|c|c|c|}
\hline & $\mathrm{Rt}_{\mathrm{IAA}}$ & $\mathrm{Rt}_{\mathrm{AA}}$ & Area $_{\text {IAA }}$ & Area $_{\mathrm{AA}}$ & Asym $_{\text {IAA }}$ & Asym $_{\mathrm{AA}}$ & $N_{\text {IAA }}$ & $N_{\mathrm{AA}}$ & Rs \\
\hline 10 & $4.49 \pm 0.01$ & $5.71 \pm 0.01$ & $193.3 \pm 0.4$ & $187.2 \pm 0.4$ & $1.05 \pm 0.01$ & $1.01 \pm 0.01$ & $464 \pm 8$ & $801 \pm 4$ & $1.50 \pm 0.01$ \\
\hline 15 & $4.45 \pm 0.01$ & $5.63 \pm 0.01$ & $194.1 \pm 0.2$ & $188.1 \pm 0.2$ & $1.03 \pm 0.01$ & $0.99 \pm 0.01$ & $516 \pm 7$ & $815 \pm 5$ & $1.50 \pm 0.01$ \\
\hline 20 & $4.44 \pm 0.02$ & $5.60 \pm 0.03$ & $192.1 \pm 0.6$ & $186.1 \pm 0.9$ & $1.00 \pm 0.02$ & $1.00 \pm 0.02$ & $583 \pm 15$ & $804 \pm 21$ & $1.52 \pm 0.04$ \\
\hline 25 & $4.40 \pm 0.01$ & $5.51 \pm 0.01$ & $194.0 \pm 0.3$ & $187.8 \pm 0.4$ & $0.98 \pm 0.01$ & $0.95 \pm 0.01$ & $570 \pm 3$ & $812 \pm 5$ & $1.48 \pm 0.01$ \\
\hline 30 & $4.36 \pm 0$ & $5.44 \pm 0.01$ & $194.4 \pm 0.4$ & $188.0 \pm 0.4$ & $0.97 \pm 0.01$ & $0.94 \pm 0.01$ & $578 \pm 6$ & $796 \pm 7$ & $1.45 \pm 0.01$ \\
\hline
\end{tabular}

Column temperature was also optimized. Increasing temperature from 10 to $30{ }^{\circ} \mathrm{C}$ resulted in a slight decrease in retention time of AA and IAA, and also a decrease in resolution (Table 4). No significant differences were observed for the peak areas of AA and IAA for the different column temperatures, and the column temperature of $20^{\circ} \mathrm{C}$ was chosen.

The injection volume was optimized and varied from 5 to $100 \mu \mathrm{L}$. It was observed that injection volumes over $10 \mu \mathrm{L}$ increased the peak asymmetry, and injections superior to $50 \mu \mathrm{L}$ resulted in badly defined peaks that ruined the resolution of IAA and $\mathrm{AA}$, and $10 \mu \mathrm{L}$ was used as the injection volume. Also the presence of a high ionic strength in the sample was assayed by adding sodium chloride to the IAA and AA standard solution until attaining $1 \mathrm{M}$ concentration, and no significant change in retention time, resolution, peak shape, and area were observed.

\section{System suitability test and robustness}

The SST was performed by ten repeated injections of a mixed solution of IAA and AA prepared in aqueous solution containing 5\% MPA, $2 \mathrm{mM}$ EDTA, and $2 \mathrm{mM}$ TCEP and was analyzed under optimum conditions. As the method was intended for the analysis of more complex matrixes than standard solutions, a cleaning $(20 \%$ of acetonitrile and $80 \%$ of $0.1 \%$ TFA during $5 \mathrm{~min}$ ), and a 7-min conditioning step to the initial conditions was introduced. Parameters such as number of theoretical plates, peak asymmetry, resolution of individual compounds, and the repeatability of reference standard solution injection were established. All parameters were in the acceptance level (Table 5), and good retention time and area repeat-

Table 5 System suitability results

\begin{tabular}{lcll}
\hline & IAA & AA & Limits \\
\hline Theorical plates & 583 & 801 & $N>200$ \\
Resolution & 1.53 & - & Rs $>1.5$ \\
Asymmetry & 1.0 & 1.0 & As $<2.0$ \\
Rt repeatability & 0.03 & 0.04 & $\mathrm{RSD}<1 \%$ \\
Area repeatability & 0.71 & 0.54 & $\mathrm{RSD}<1 \%$ \\
\hline
\end{tabular}

ability was observed. None of the aqueous solution components did cause any problems in the retention time stability, baseline stability, and column lifetime, and no interfering peaks due to these components were observed in the time range of the IAA and AA retention times. Over 600 injections containing these three components were performed on the same column without any change in retention time, backpressure, and efficiency, showing that the TSKgel Amide- 80 stationary phase is compatible with the injection of the $100 \%$ aqueous extraction solution employed for maximizing the AA and IAA stability and simultaneous reduction of IDHA and DHA to IAA and AA, a key step for performing total AA and IAA quantification.

The robustness of the proposed HILIC method was tested by deliberately introducing small changes in the method, namely percentage of organic solvent $(89 \%$ and $91 \%)$, percentage of TFA in the aqueous solution $(0.9 \%$ and $1.1 \%)$, column temperature $\left(17\right.$ and $\left.23{ }^{\circ} \mathrm{C}\right)$, and mobile phase flow rate $(0.6$ and $0.8 \mathrm{~mL} / \mathrm{min})$ and evaluating the same method parameters as determined for the SST. Temperature, flow rate, and acetonitrile percentage showed significant effects on retention time of IAA (Fig. 2a) and AA (Fig. 2b). The TFA percentage on the aqueous phase did not show any significant effect on retention time of AA and IAA and in neither of the other studied chromatographic parameters. Temperature was the factor that had a lower influence on retention time (only 0.00764 and $0.014 \mathrm{~min} /{ }^{\circ} \mathrm{C}$, for IAA and $\mathrm{AA}$, respectively), and the observed effect for a $4{ }^{\circ} \mathrm{C}$ variation is still within the acceptable limits for retention time repeatability. On the other hand, a $0.1 \mathrm{~mL} / \mathrm{min}$ variation in the flow rate introduces a change of $0.643 \mathrm{~min}$ for IAA and $0.808 \mathrm{~min}$ for AA, and for the acetonitrile percentage, a $1 \%$ variation introduces a change in $0.298 \mathrm{~min}$ for IAA and $0.505 \mathrm{~min}$ for AA. For resolution, only the acetonitrile percentage showed a significant effect; nevertheless, only for the $89 \%$ of acetonitrile was the resolution lower than the 1.5 limit (Fig. 2c). Peak asymmetry was significantly influenced only by acetonitrile percentage and flow rate, but all values obtained were within the 2.0 limit (Fig. 2d). The efficiency was significantly influenced by all factors but the percentage of TFA, but all values were above the 200 plate cut-off 
a

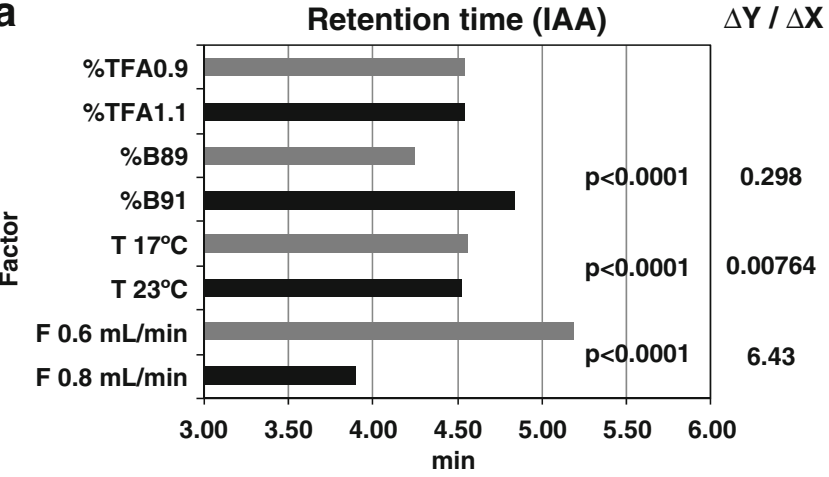

C

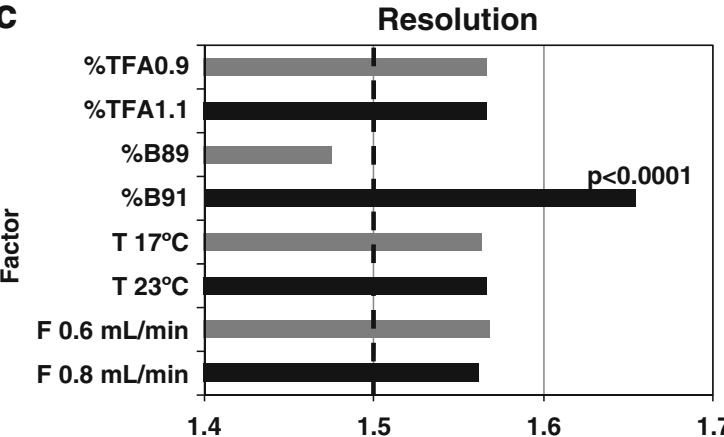

e
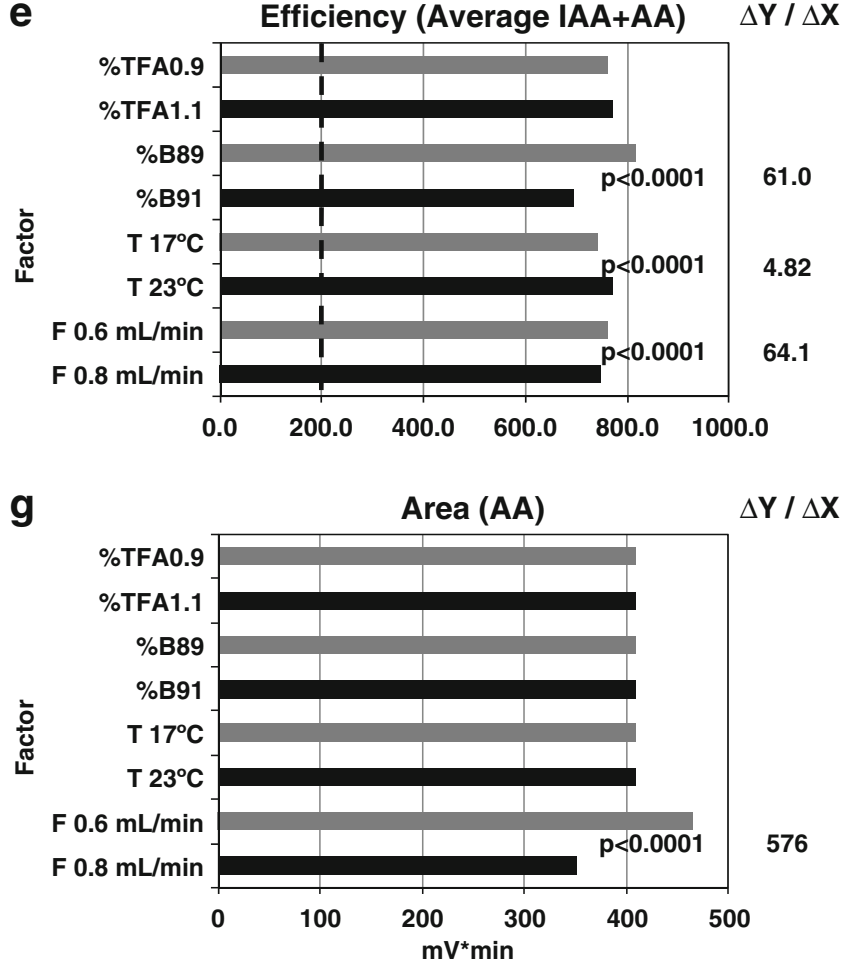

b

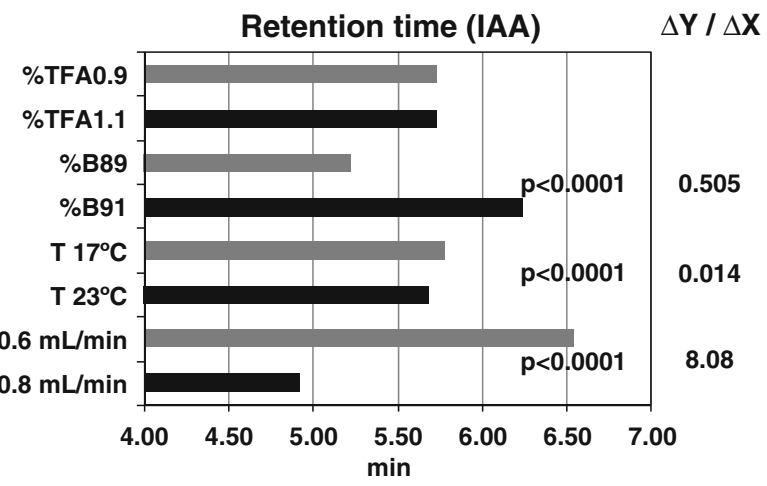

d

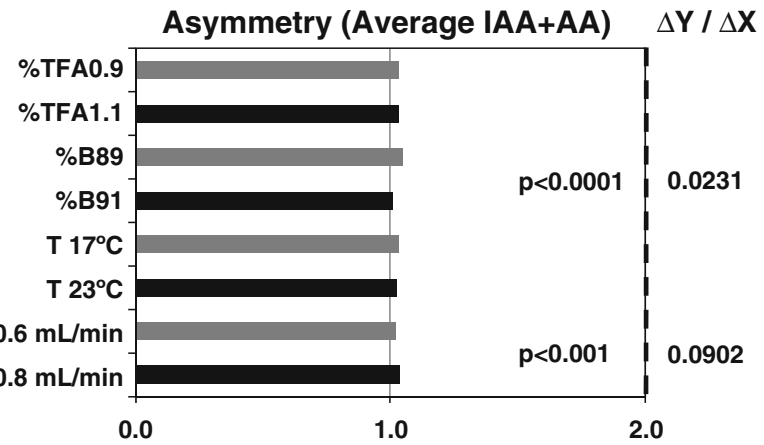

f

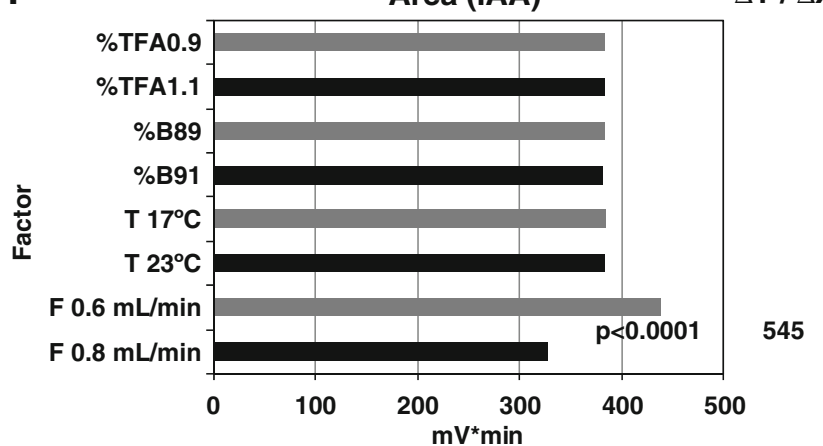

h

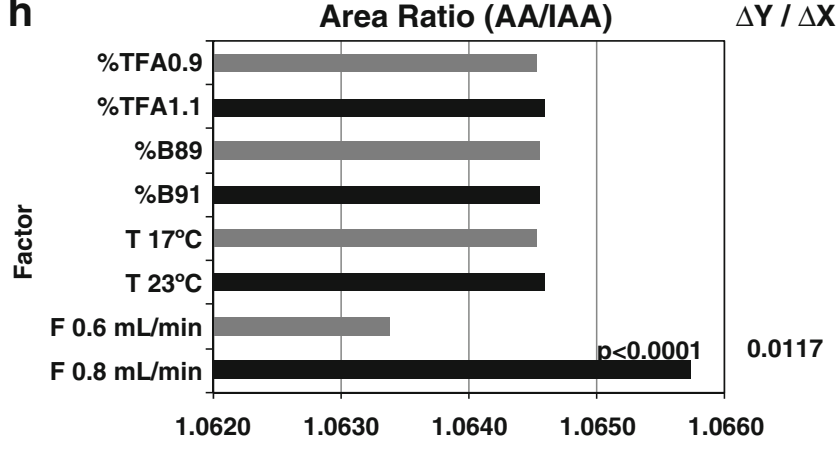

Fig. 2 Robustness results for the chromatographic parameters of the separation of IAA and AA

(Fig. 2e). For IAA and AA area and AA/IAA area ratio, only the flow rate showed a significant effect (Fig. $2 \mathrm{f}-\mathrm{h}$ ). For IAA and AA area, a $0.1 \mathrm{~mL} / \mathrm{min}$ change introduces a $14 \%$ area change, well above the area repeatability limit, but for the area ratio, the change observed is only $1.7 \%$, and although higher than the limits, it is lower than the change observed for the AA area alone. The critical parameters in the HILIC chromatographic separation of AA and IAA are flow rate and acetonitrile percentage, and these should not vary more than $\pm 0.007 \mathrm{~mL} / \mathrm{min}$ for maintaining the area and also 


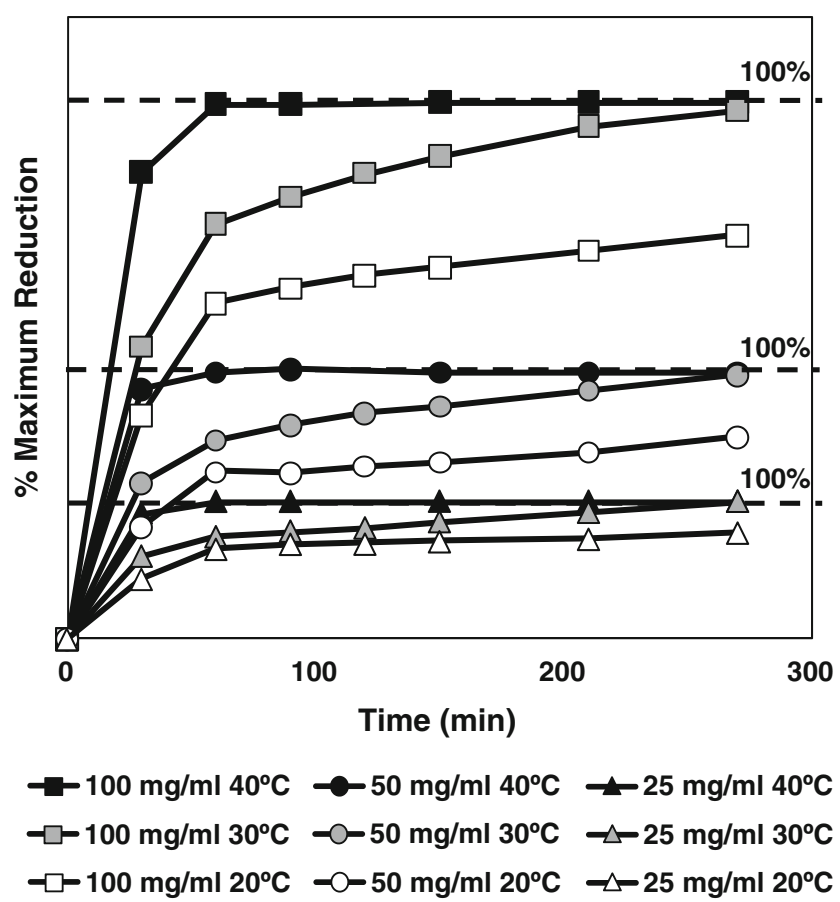

Fig. 3 Kinetics of TCEP reduction of DHA at different concentration and temperatures

retention time repeatability within the $1 \%$ tolerance, and the percentage of acetonitrile should be allowed a variation not more than $\pm 0.15 \%$ in order to maintain the retention time repeatability and resolution, although for this late parameter, a tolerance of $\pm 0.7 \%$ of acetonitrile will result in a resolution within the acceptable value. In many of the parameters studied, there was a significant interaction between the factors, but the variations resulting from these interactions were much lower than that observed for the individual variables, and so they were not taken into account.
DHA and IDHA reduction recovery and IAA and AA stability evaluation

A critical step in a successful quantification of total IAA and $\mathrm{AA}$ is the quantitative reduction of IDHA and DHA present in the sample back to IAA and AA and also avoiding their degradation during the extraction and analysis step. For IAA and AA stabilization and IDHA and DHA reduction MPA at 5\% $(w / v)$, EDTA and TCEP at $2 \mathrm{mM}$ were used $[13-15,21,22,27]$. Although the reduction of IDHA and DHA with TCEP is faster at neutral to low acidic $\mathrm{pH}$ [27], it can also be performed at $\mathrm{pH} 2$ [28] where the stability of AA, IAA, and also TCEP are maximum. In order to optimize the reduction conditions, as no data are available for such a high MPA concentration (5\%), the DHA was reduced with TCEP at three different concentrations $(100,50$, and $25 \mathrm{mg} / \mathrm{L})$ and three different reduction temperatures $\left(40,30\right.$, and $\left.20{ }^{\circ} \mathrm{C}\right)$. At $40{ }^{\circ} \mathrm{C}$ for the three concentrations tested, the reduction of DHA reached almost $100 \%$ in $1 \mathrm{~h}$ (Fig. 3). At $30{ }^{\circ} \mathrm{C}$ in average, $99 \%$ reduction was only achieved after $4.5 \mathrm{~h}$, and for $20^{\circ} \mathrm{C}$, only an average of $76 \%$ of maximum reduction was achieved in the same time period. For the three concentrations studied, the AA was stable for $3.5 \mathrm{~h}$ at $40^{\circ} \mathrm{C}$, as the concentration of AA remained constant. Nevertheless in all cases, only in average of $65 \%$ of the theoretical AA concentration was recovered by the DHA reduction. In order to test the DHA and IDHA stability under the reduction conditions applied, IDHA was generated from IAA by adding IAA $(500 \mathrm{mg} / \mathrm{L})$ to DHA $(1,000 \mathrm{mg} / \mathrm{L})$ in water. During the first $60 \mathrm{~min}$, there was a pseudo-zero order decrease in the amount of IAA that was closely followed by an increase in the amount of AA, leading to a decrease of $42 \mathrm{mg} / \mathrm{L}$ of IAA (Fig. 4). When the reduction solution was added and the mixture was heated at $40{ }^{\circ} \mathrm{C}$,
Fig. 4 Recovery of IAA and AA after incubation in water at room temperature followed by reduction with TCEP

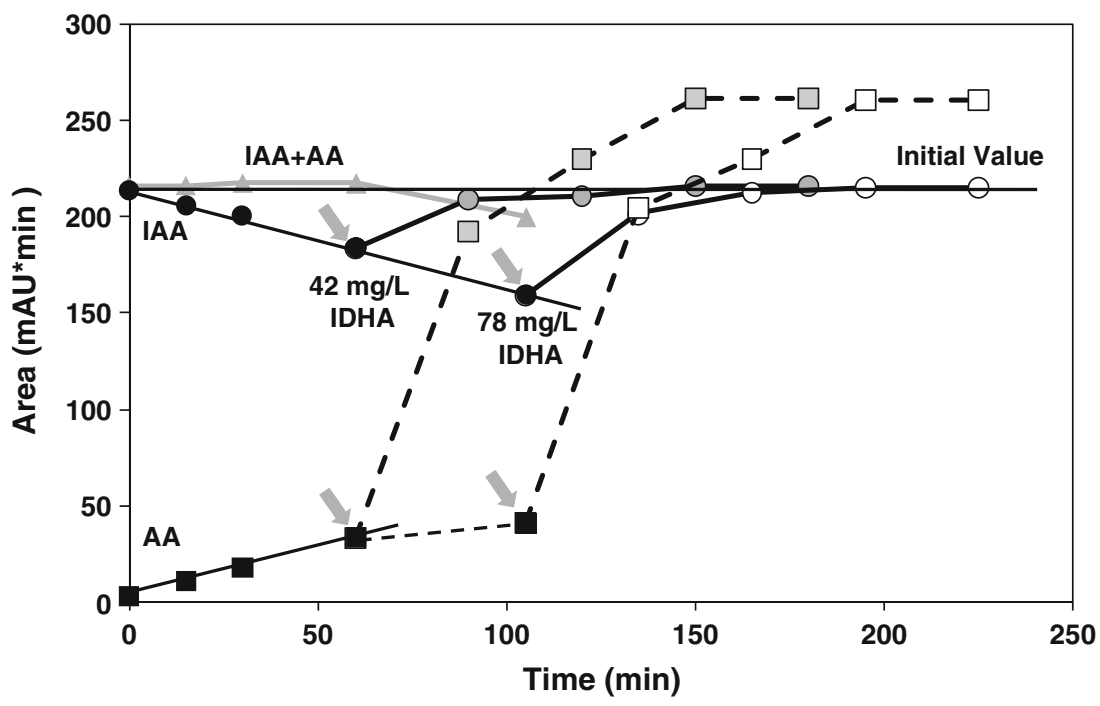


Table 6 Detection of systematic errors by standard addition calibration and Youden calibration for the food matrixes studied $(y=a x+b)$

$E C$ external calibration, ISC internal standard calibration, $S A C$ standard addition calibration, $Y C$ Youden calibration

\begin{tabular}{lllll}
\hline & $a$ & $b$ & $r$ & $t$ value \\
\hline AA & & & & \\
EC & $0.7775 \pm 0.0006608$ & $0.2061 \pm 0.1365$ & 0.99995 & \\
ISC & $3.982 \times 10^{-3} \pm 3.196 \times 10^{-6}$ & $0.001345 \pm 0.0006318$ & 1.000 & \\
Orange juice-external calibration & & & \\
SAC & $0.7899 \pm 0.008449$ & $49.85 \pm 3.405$ & 0.9994 & $1.463(p<0.150)$ \\
YC & $43.73 \pm 2.385$ & $0.7855 \pm 1.338$ & 0.9941 & $0.5893(p<0.572)$ \\
Chestnut—external calibration & & & \\
SAC & $0.7614 \pm 0.009784$ & $45.22 \pm 4.191$ & 0.9989 & $1.642(p<0.107)$ \\
YC & $49.69 \pm 1.032$ & $1.069 \pm 0.5375$ & 0.9994 & $1.989(p<0.0819)$ \\
Chestnut-internal standard calibration & & & \\
SAC & $3.930 \times 10^{-3} \pm 3.653 \times 10^{-5}$ & $0.2663 \pm 0.01331$ & 0.9997 & $1.465(p<0.150)$ \\
YC & $0.3583 \pm 0.007028$ & $0.003988 \pm 0.003660$ & 0.9994 & $1.090(p<0.308)$ \\
IAA & & & & \\
EC & $0.7820 \pm 0.001182$ & $0.4934 \pm 0.2469$ & 0.99991 & \\
Ham external calibration & & & \\
SAC & $0.7726 \pm 0.006306$ & $31.24 \pm 1.722$ & 0.9997 & $1.429(p<0.159)$ \\
YC & $60.95 \pm 0.6783$ & $0.7000 \pm 0.3826$ & 0.9997 & $1.830(p<0.105)$ \\
\hline
\end{tabular}

the amount of IAA was recovered completely after $60 \mathrm{~min}$. The amount of AA recovered reached the maximum value also after $90 \mathrm{~min}$, and again, the value recovered was only nearly $65 \%$ of the theoretical yield. After standing $105 \mathrm{~min}$ in water, the amount of IAA converted to IDHA was $78 \mathrm{mg} /$ $\mathrm{L}$, and again when the reduction solution was added and the mixture was heated at $40{ }^{\circ} \mathrm{C}$, the amount of IAA recovered to the initial value, and the AA amount recovered was the same as that observed in the first $60 \mathrm{~min}$ (Fig. 4). These results show that IDHA and DHA are stable under the condition used for reduction, and the purity of the commercial DHA used was only nearly $65 \%$.

The stability of IAA and AA was evaluated during the waiting phase between sample preparation and analysis by HPLC. Most published methods advise the use of a refrigerated autosampler at $4{ }^{\circ} \mathrm{C}$ to prevent AA degradation during the waiting step before analysis [13-15, 21]. The stability of IAA and AA at three different concentrations $(\sim 250,100$, and $25 \mathrm{mg} / \mathrm{L})$ were evaluated during a 48$\mathrm{h}$ period at room temperature and at $4{ }^{\circ} \mathrm{C}$. Whatever the concentration, the IAA and AA were stable during the 48$\mathrm{h}$ period studied, and there was no significant difference between the AA and IAA stability at room temperature and $4{ }^{\circ} \mathrm{C}$, showing that the stabilization-extraction solution was able to maintain the levels of IAA and AA at room temperature during a normal period of HPLC analysis. Nevertheless, after 1 week of storage at room temperature, there was a $30 \%$ reduction on the amount of AA and IAA, and at $4{ }^{\circ} \mathrm{C}$, the amount of $\mathrm{AA}$ and IAA remained constant; therefore for longer storage periods, the refrigeration temperature is advisable.
In-house method validation

The in-house method validation was performed for two different quantification situations, external calibration for determining AA and IAA and internal standard calibration for quantification of AA using IAA as internal standard. The matrixes assayed were fruit juices (pear, mango, and orange juices - results only shown for orange juice), freezedried chestnuts, and ham, and for internal standard calibration, only freeze-dried chestnuts were assayed, as preliminary runs showed that for ham, AA was not a suitable internal standard as it was present in the sample. The method was found to be linear in the 1.0 to $500 \mathrm{mg} / \mathrm{L}$ range for both IAA and AA external calibration and also for AA internal standard calibration. The detection and quantification limit for AA and IAA were low enough (LOQ 1.5 and $3.7 \mathrm{mg} / \mathrm{L}$ for AA and IAA, respectively) to be applied to a range of food matrixes besides those studied by us. Method precision and recovery were tested in each sample matrix by the external calibration and in chestnuts for the internal standard calibration. A typical chromatogram for each sample studied is shown in Fig. 5. Repeatability and intermediate precision figures were similar for the determination of AA in orange juice and chestnuts and IAA in ham and ranged from $1.43 \%$ to $1.92 \%$ and $2.59 \%$ to $3.01 \%$, respectively. The internal standard approach yielded better repeatability and intermediate precision values $(0.87 \%$ and $1.73 \%$, respectively) for the chestnut sample. These values are within acceptable limits and in accordance with the Horwitz criterion [43, 44] for the range of values present in the analyzed samples. 

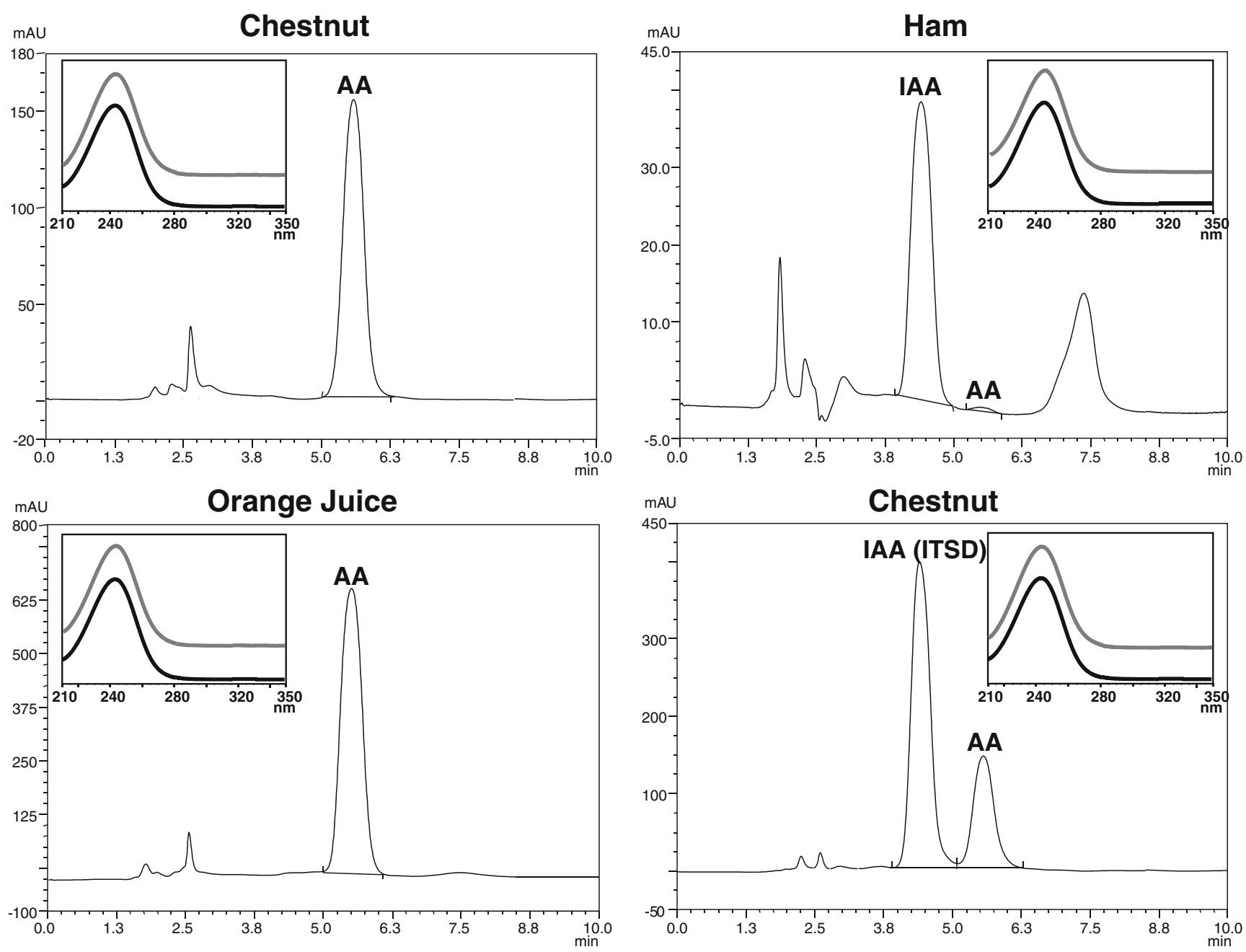

Fig. 5 Typical chromatograms and UV spectrum obtained for the analysis of the tested food matrixes on TSKgel Amide-80. Sample UV spectrum (gray lines), standard UV spectrum (black lines)

Recovery values were similar for the three different spiking levels $(25,250$, and $500 \mathrm{mg} / \mathrm{L})$ for AA and IAA and ranged from $96.41 \%$ to $103.49 \%$. Similar values were obtained for the AA determination in chestnuts by internal standard calibration $(96.8 \%$ to $103.93 \%)$. All recovery values were within the acceptable limits [45].

The influence of the different matrixes on AA and IAA response was evaluated by standard addition calibration and Youden calibration for detecting proportional and constant bias, respectively [40, 41]. For all matrixes tested, the slope obtained by the standard addition calibration was not significantly different from that obtained by external and internal standard calibration (Table 6). For the Youden calibration, the intercept obtained was not significantly different from zero (Table 6), showing no evidence of proportional or constant bias in the studied matrixes.

An excellent match between the real samples UV spectrums and that of pure AA and IAA standards were obtained (Fig. 5). Also the peak purity parameters built in the Chromeleon software were used to evaluate possible interfering co-eluting materials and peak homogeneity. The peak purity match factor that measures the UV spectrum homogeneity in the peak were between 999 and 1,000 for both AA and IAA in the samples analyzed.

\section{Method applicability}

Besides the vitamin $\mathrm{C}$ determination (Total AA + DHA), there is sometimes needed to measure separately the content of AA and DHA in a sample. The common approach is to perform a duplicate determination of the sample with and without reducing agent, and the amount of $\mathrm{AA}$ is determined directly on the sample without reduction and the DHA content by difference between the AA level in the reduced and unreduced sample solution $[15,27]$. Due to the excellent compatibility with the ordinary extractionstabilizing reducing solution, the present HILIC method can be also applied to determine the DHA and AA by the 
traditional subtraction method. The conditions employed for sample stabilization without reducing agent must be controlled in order to prevent the conversion of AA to DHA $[15,27]$, and this involves the temperature control $\left(4{ }^{\circ} \mathrm{C}\right)$ during all analytical operations and in the autosampler. The stability of the AA and IAA without reducing agent during the chromatographic separation was tested, and no significant difference was observed between the peak area of the $\mathrm{AA}$ and IAA in the reducing solution (stored at room temperature) and in a solution containing only 5\% MPA and $2 \mathrm{mM}$ EDTA solution (stored at $4{ }^{\circ} \mathrm{C}$ ).

The HILIC method was also applied to an acetylsalicylic acid analgesic with vitamin $\mathrm{C}$ and to a multivitamin tablet. The values found were $238.93 \pm 2.86$ and $61.05 \pm 1.22 \mathrm{mg} /$ tablet, respectively, which were in good agreement with the declared values of 240 and $60 \mathrm{mg} /$ tablet. The selectivity of the method for these applications was confirmed by examination of the UV spectrum, which was identical to the AA standard.

Although only AA quantification by internal standard calibration was validated, the quantification of IAA by internal standard calibration can also be performed, except in the case of the presence in the original sample of AA, as observed for the ham sample (Fig. 5).

\section{Conclusions}

The proposed HILIC method allows a fast, simple, flexible, and robust alternative to conventional ion-pair and RP C18 , enabling baseline resolution of AA and IAA in a short time. The TSKgel Amide- 80 column is compatible with injections of high concentrations of MPA without drift of baseline and retention time, allowing sample preparation in other solutions than the mobile phase used for the HILIC as noted in the previous HILIC approaches. This broadens the range of stabilization options, as the one used by us, which was found suitable for routine applications. The use of an extraction-reduction solution containing MPA, TCEP, and EDTA and a reduction step at $40{ }^{\circ} \mathrm{C}$ allows in one step and with the use of a single solution, the extraction and quantitative conversion of DHA and IDHA to AA and IAA, respectively. AA and IAA were stable for at least $48 \mathrm{~h}$ at room temperature, without the need to degas samples and the use of special glassware. One of the main advantages of the described HILIC method is simplicity and flexibility, as no complicated mobile phase mixtures containing ion-pair reagents or inorganic buffers were needed. The retention time of AA and IAA could be adjusted very easily according to the needs by simple changing of the ratio of aqueous and organic content of the mobile phase. The use of gradient elution is also a possibility if needed. The use of a short column and a high percentage of acetonitrile allow using high mobile phase flow rates with low backpressures, being possible to resolve AA and IAA in nearly $2 \mathrm{~min}$.

The method is reliable for both quantification of AA and IAA by external calibration and AA by internal standard calibration. In this last method, IAA was used as internal standard, due to the fact that it is an epimer of AA and is an excellent internal standard, as with the exception of ascorbate oxidase activity, the other parameters influencing the AA stability and reducing efficiency affect similarly the IAA. The use of an internal standard also simplifies the extraction-reduction protocol.

Acknowledgments The authors gratefully acknowledge the financial support provided by the Research Unit Centro de Química de Vila Real (POCTI-SFA-3-616) and FCT (Foundation for Science and Technology).

\section{References}

1. Berger TM, Polidori MC, Dabbagh A, Evans PJ, Halliwell B, Morrow JD, Roberts LJ II, Frei B (1997) J Biol Chem 272:15656

2. Packer L (1997) Vitamin C in health and disease. CRC, Boca Raton

3. Davey MW, Van Montagu M, Inze D, Sanmartin M, Kanellis A, Smirnoff N et al (2000) J Sci. Food Agric 80:825

4. Rumsey SC, Levine M (1998) J Nutr Biochem 9:116

5. Wells WW, Xu DP, Washburn MP (1995) Meth Enzymol 252:30

6. Directive 90/496/EEC on nutrition labelling for foodstuffs

7. Directive $95 / 2 / \mathrm{EC}$ on food additives other than colours and sweeteners

8. Fabianek J, Herp A (1967) Proc Soc Exp Biol Med 125:462

9. AOAC Official Method 967.21 (2000) Official Methods of Analysis of AOAC International/Dr Willian Horwitz, 17. ed. AOAC International, Maryland

10. AOAC Official Method 984.26 (2000) Official Methods of Analysis of AOAC International/Dr Willian Horwitz, 17. ed. AOAC International, Maryland

11. Eitenmillar RR, Landen WO (2000) Vitamin analysis for the health and food sciences. CRC, Boca Raton, pp 223-270

12. Arya SP, Mahajan M, Jain P (2000) Anal Chim Acta 417:1

13. Washko PW, Welch RW, Dhariwal KR, Wang Y, Levine M (1992) Anal Biochem 204:1

14. Nováková L, Solich P, Solichová D (2008) Trends Anal Chem 27:942

15. Ali MS, Phillippo ET (1996) J AOAC Int 79:803

16. Kall MA, Andersen C (1999) J Chromatogr B 730:101

17. Nyyssonen K, Salonen JT, Parviainen MT (2000) In: De Leenheer AP, Lambert WE, Van Bocxlaer JF (eds) Modern chromatographic analysis of vitamins, 3rd ed. Marcel Dekker, New York, pp 271300

18. Lykkesfeldt J (2000) Anal Biochem 282:89

19. Wechtersbach L, Cigić B (2007) J Biochem Biophys Methods 70:767

20. Brause R, Woollard DC, Indyk HE (2003) J AOAC Int 86:367

21. Behrens W, Madere R (1994) J Liq Chromatogr 17:2445

22. Vanderslice JT, Higgs DJ (1984) J Chromatogr Sci 22:485

23. Lykkesfeldt J, Loft S, Poulsen HE (1995) Anal Biochem 229:329

24. Schell DA, Bode AM (1993) Biomed Chromatogr 7:267

25. Iwase H (1992) J Chromatogr 606:277

26. Lykkesfeldt J (2000) Current protocols in toxicology, Unit 7.6. Wiley, New York 
27. Donner LW, Hicks KB (1981) Anal Chem 115:225

28. Margolis SA, Schapira RM (1997) J Chromatogr B 690:25

29. Rose RC, Koch MJ (1984) Anal Biochem 143:21

30. Tai A, Gohda E (2007) J Chromatogr B 853:214

31. Nováková L, Solichová D, Pavlovicová S, Solich P (2008) J Sep Sci 31:1634

32. Karatapanis AE, Fiamegos YC, Stalikas CD (2009) J Sep Sci 32:909

33. Nováková L, Solichová D, Solich P (2009) J Chromatogr A 21:4574

34. Guo Y, Gaiki S (2005) J Chromatog A 1074:71

35. Yoshida T (1997) Anal Chem 69:3038-3043

36. Thompson M, Ellison S, Wood R (2002) Pure Appl Chem 74:835
37. European Pharmacopoeia (Ph. Eur. 5) (2004) Council of Europe, Strasbourg, 5 th ed

38. ISO (1990) ISO 8466-1. ISO, Geneva

39. Currie LA (1995) Pure Appl Chem 67:1699

40. Campaña AMG, Rodríguez LC, Muñoz JA, Barrero FA, Ceba MR, Baeyens WRG (1999) Biomed Chromatogr 13:151

41. Rodríguez LC, Gracia LG, López EMA, Sendra JMB (2001) Trends Anal Chem 20:620

42. Dejaegher B, Mangelings D, Heyden YV (2008) J Sep Sci 31:1438

43. Horwitz W (1982) Anal Chem 54:67

44. Thompson M (2000) Analyst 125:385

45. Taverniers I, De Loose M, Van Bockstaele E (2004) Trends Anal Chem 23:535 